\title{
Spatial variation of eddy-diffusion coefficients in the turbulent plasma sheet during substorms
}

\author{
M. Stepanova1 ${ }^{1}$, E. E. Antonova ${ }^{2,3}$, D. Paredes-Davis ${ }^{1}$, I. L. Ovchinnikov ${ }^{2}$, and Y. I. Yermolaev ${ }^{3}$ \\ ${ }^{1}$ Physics Department, Universidad de Santiago de Chile, Chile \\ ${ }^{2}$ Sobeltsyn Institute of Nuclear Physics, Moscow State University, Russia \\ ${ }^{3}$ Space Research Institute, RAS, Russia
}

Received: 22 September 2008 - Revised: 26 February 2009 - Accepted: 28 February 2009 - Published: 1 April 2009

\begin{abstract}
Study of the plasma turbulence in the central plasma sheet was performed using the Interball-Tail satellite data. Fluctuations of the plasma bulk velocity in the plasma sheet were deduced from the measurements taken by the Corall instrument for different levels of geomagnetic activity and different locations inside the plasma sheet. The events that satisfied the following criteria were selected for analysis: number density $0.1-10 \mathrm{~cm}^{-3}$, ion temperature $T \geq 0.3 \mathrm{keV}$, and average bulk velocity $\leq 100 \mathrm{~km} / \mathrm{s}$. It was found that the plasma sheet flow generally appears to be strongly turbulent, i.e. is dominated by fluctuations that are unpredictable. Corresponding eddy-diffusion coefficients in Y- and Z-direction in the GSM coordinate system were derived using the autocorrelation time and rms velocity. Statistical studies of variation of the eddy-diffusion coefficients with the location inside the plasma sheet showed a significant increase in these coefficients in the tailward direction. During substorms this dependence shows strong increase of eddy-diffusion in the central part of the plasma sheet at the distances of 10-30 Earth's radii. This effect is much stronger for Y-components of the eddy-diffusion coefficient, which could be related to the geometry of the plasma sheet, allowing more room for development of eddies in this direction.
\end{abstract}

Keywords. Magnetospheric physics (Magnetospheric configuration and dynamics; Plasma sheet; Storms and substorms)

\section{Introduction}

The magnetosphere of the Earth is formed as a result of an interaction between the solar wind supersonic and superalfvenic turbulent flow and the geomagnetic field. It is

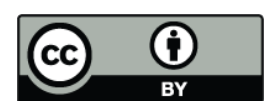

Correspondence to: $\mathrm{M}$. Stepanova

(marina.stepanova@usach.cl) well known that turbulence in ordinary fluids significantly changes the basic properties of flow and large-scale flow patterns, therefore the turbulent processes should also play an important role in the dynamics of the Earth's magnetosphere. For example, Borovsky and Funsten (2003a); Borovsky (2005) studied the turbulence effect in the solarwind/magnetosphere coupling; they have found that the geomagnetic activity is greater when the solar wind turbulence is louder.

In this work we assume that the magnetosphere is formed as a turbulent wake behind the Earth. However, there is a significant difference between a turbulent wake in a fluid behind an ordinary obstacle and the geomagnetic tail (magnetotail). The cross section radius of the ordinary wake is close to the obstacle size. On the contrary, extended over hundreds of Earth's Radii $\left(R_{E}\right)$, the Earth's magnetotail is separated into the plasma sheet, and the tail lobes.

Antonova and Ovchinnikov (1996, 1999) proposed that comparatively stable turbulent plasma sheet can be formed when the regular plasma transport related to the dawn-dusk electric field across the plasma sheet is compensated by the turbulent transport via eddy diffusion. Eddy diffusion characterizes the plasma transport under the conditions of turbulent convection (Borovsky et al., 1998). Antonova and Ovchinnikov $(1996,1999)$ consider that the integral particle flux is equal to $\mathbf{J}=n\langle\mathbf{V}\rangle-D n$, where $n$ is the averaged over the turbulent fluctuations plasma particle density, $\langle V\rangle$ is the averaged bulk velocity, $D$ is the eddy diffusion coefficient derived using the autocorrelation time and rms velocity. When the turbulent fluctuations act to expand the plasma sheet, the large-scale electrostatic dawn-dusk electric field counteracts to compress it, similarly to the case of the laboratory pinch which is compressed by the induction electric field. When the expansion and compression compensate each other, a stationary structure is formed. This assumption allows to predict the order of magnitude of the eddy diffusion coefficient in Z-direction $\sim 10^{5}-10^{6} \mathrm{~km}^{2} / \mathrm{s}$, which

Published by Copernicus Publications on behalf of the European Geosciences Union. 
is necessary to reproduce the observed plasma sheet thickness. This value agrees with the values of the eddy-diffusion coefficients, obtained using the ISEE-2, Interball/Tail, and GEOTAIL satellite data (Borovsky et al., 1998; Borovsky and Funsten, 2003b; Ovchinnikov et al., 2000; Troshichev et al., 2002; Stepanova et al., 2005). Proposed mechanism also explains the appearance of theta-aurora observed during the periods of the prolonged existence of the northward-oriented interplanetary magnetic field (IMF) when the tail lobes are filled by the plasma sheet plasma (Antonova and Ovchinnikov, 2001).

The presence of geomagnetic substorms affects the plasma sheet turbulence: Neagu et al. $(2002,2005)$ have shown using the ISEE-2 and the AMPTE/IRM satellite measurements that both velocity and especially geomagnetic field fluctuations increase with the auroral electrojet (AE) index. It was also found, that level of these fluctuations correlates with the solar activity. Ovchinnikov et al. (2000) and Stepanova et al. (2005) analyzed a relationship between the values of eddy-diffusion coefficient in the plasma sheet and the phases of isolated geomagnetic substorms, using the Interball/Tail satellite data. They have found that the values of eddy-diffusion coefficient increase significantly during the growth and especially expansion phases. Stepanova et al. (2005) also showed that during these phases the values of the eddy-diffusion coefficient become stronger dependent on the strength of the geomagnetic field B. Shiokawa et al. (2005) investigated the magnetic field fluctuations during the substorm-associated dipolarizations in the night-side plasma sheet using the Geotail satellite data. They found that the spectral shape of the magnetic field fluctuations depends on the absolute value of the magnetic field, suggesting possible transition from a three-dimensional turbulence in smaller Bregion to a two-dimensional turbulence in a larger B-region.

Despite significant achievements in understanding of the turbulent processes in the plasma sheet, many questions still remain unanswered. In this work we study the spatial variation in the eddy diffusion coefficients inside the plasma sheet during quiet intervals and different phases of geomagnetic substorms.

\section{Instrumentation and data analysis}

For this study we used INTERBALL/TAIL probe data accumulated when the satellite orbit was crossing the plasma sheet between 6 and 30 Earth's radii. In particular we used the moments of ion distribution functions obtained using the data of the hemispherical electrostatic ion energy spectrometer (CORALL) in the range from 30 to $24200 \mathrm{eV} / \mathrm{q}$ (Yermolaev et al., 1997). The satellite spin axes was oriented along the X-direction of the GSM coordinate system, hence the ion bulk velocities were obtained in Y- and Z-directions only. We also used $2 \mathrm{~min}$ averaged geomagnetic field data, obtained by a digital fluxgate magnetometer MIF-M/PRAM
(Klimov et al., 1997). Only the events that satisfied the following criteria were selected for analysis: number density $0.1-10 \mathrm{~cm}^{-3}$, ion temperature $T \geq 0.3 \mathrm{keV}$, and average bulk velocity $\leq 100 \mathrm{~km} / \mathrm{s}$. We also restricted the satellite coordinates taking into account a plasma sheet flapping motion. Specifically, we considered the following range of satellite $\mathrm{Z}$ coordinates $-8 \leq Z \leq 8 R_{E}$, and the range of the X- and Ycoordinates as shown in Fig. 1. In order to accumulate the statistically significant amount of data, we made these data selection criteria weaker than ones typically used. Thus we reached a compromise between the necessity to include an amount of data to do a reasonable statistic analysis and the necessity to avoid the data from other regions of the magnetosphere.

To obtain the eddy-diffusion coefficient we first calculated the autocorrelation function of the bulk flow velocities as in (Borovsky et al., 1997, 1998),

$A_{v}(\tau)=\frac{\sum(V(i)-\langle V\rangle) \sum(V(i+k)-\langle V\rangle)}{\sum(V(i)-\langle V\rangle)^{2}}$,

where $V$ represent either Y- or Z-components of the bulk velocity in the GSM coordinate system. Each velocity component was calculated using ion distribution functions, obtained once per each satellite rotation period $(T=120 \mathrm{~s}), \tau=k T$ is the time delay in seconds, $\langle V\rangle=\sum V(i) / N$ is the mean bulk velocity, and $N$ is the number of points used.

The autocorrelation time ( $\tau_{\text {auto }}$ ) was determined as the best fit of the natural logarithm of the autocorrelation function $\left(A(\tau)=\exp \left(-\tau / \tau_{\text {auto }}\right)\right)$ by a linear function $y=a x$, taking into account that $\tau=-1 / a$, and the error in the autocorrelation time $\Delta \tau=\Delta a / a^{2}$, where $\Delta a$ is the error of linear fit coefficient. We discarded all events with the unusually long autocorrelation times, $\tau>10 \mathrm{~min}$, since our data did not allow to resolve them properly. The algorithm for the computation of $\tau$ was optimized to minimize the error (when the number of points to fit was $\geq 3$ ). The root mean squared (rms) velocity was determined as

$V_{\mathrm{rms}}=\sqrt{\frac{\sum(V(i)-\langle V\rangle)^{2}}{N}}$

The eddy-diffusion coefficient was obtained as (Borovsky et al., 1998)

$D=\frac{V_{\mathrm{rms}}^{2} \tau_{\text {auto }}}{2}$

Autocorrelation times, rms velocities, and the resulting eddydiffusion coefficients were determined using 20 bulk velocity points, i.e. every $40 \mathrm{~min}$. This number of points does not allow to determine the $V_{\text {rms }}$ and $\tau_{\text {auto }}$ with very high accuracy, but such length of averaging interval is necessary to guarantee a reasonable space resolution. To determine whether the eddy diffusion coefficient is taken during the quiet time, expansion, or recovery phase of the substorm, we perform an analysis of the one minute resolution AL index. We average 


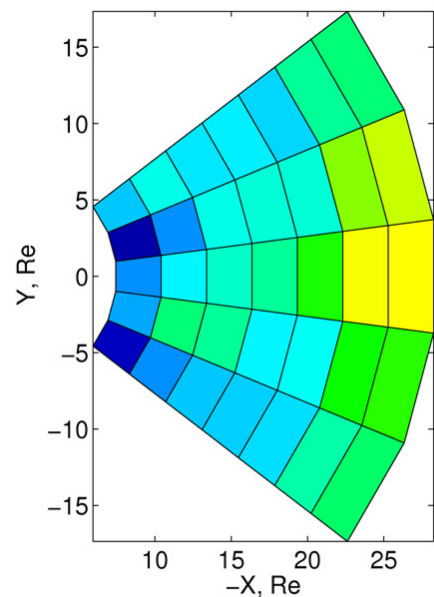

(a) $D_{y y}$, quiet

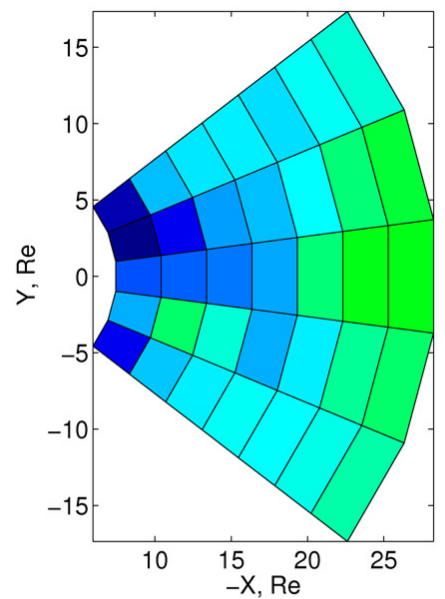

(d) $D_{z z}$, quiet

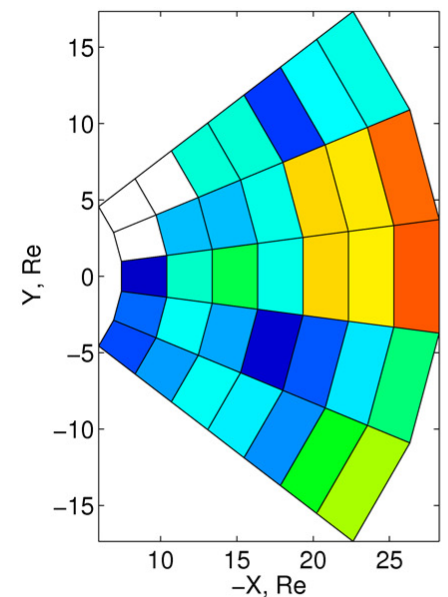

(b) $D_{y y}$, expansion

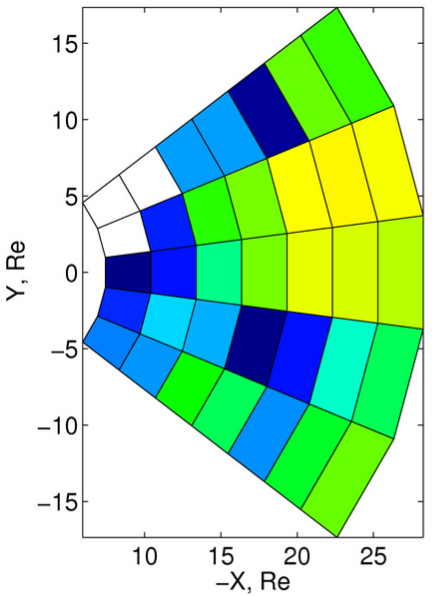

(e) $D_{z z}$, expansion

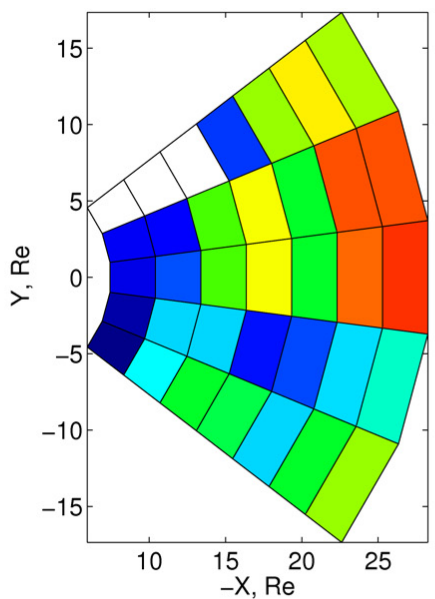

(c) $D_{y y}$, recovery

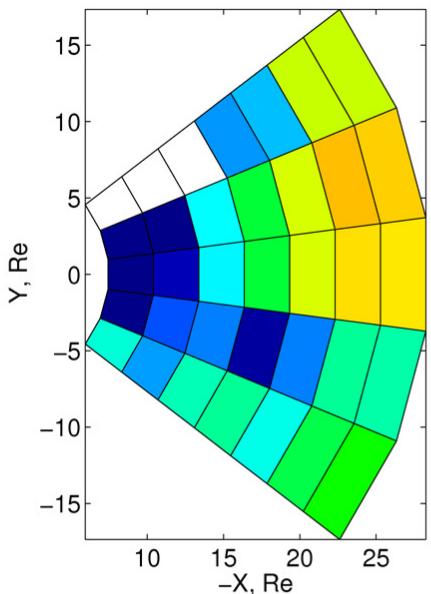

(f) $D_{z z}$, recovery

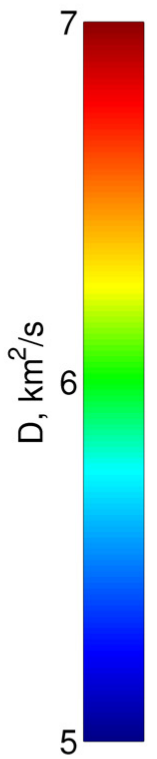

Fig. 1. Variation of eddy diffusion coefficients with the phase of geomagnetic substorms.

the 40 data points of the AL index which lie in the same temporal interval as is used for eddy diffusion calculation. In addition, we perform a linear fit to those $40 \mathrm{AL}$ index points. If the absolute value of the slope $s$ of the linear fit is below $1 / 2$ $(|s|<1 / 2)$, the event is considered to be quiet. Conversely, if $\mathrm{AL}$ index is below $-100 \mathrm{nT}$ and the value of the slope is less than $-1 / 2(s<-1 / 2)$, the event is considered to belong to a substorm in its expansion phase. Finally, AL $<-100 \mathrm{nT}$ and $s>1 / 2$ corresponds to the recovery phase of a substorm.

In this study we could not distinguish the data corresponding to the growth phase, as it was difficult to establish the clear criteria for automatic data extraction. Therefore, the majority of eddy diffusion values corresponding to the growth phase have been included into the quiet time data set. Table 1. summarizes the resulting total number of eddy diffusion values.
To analyze the spacial distribution of eddy diffusion coefficient, we perform the following procedure: The data of each set were separated into 30 overlapped bins such that: the GSM coordinates were transformed into polar ones, the radial limits were 6 and 30 Earth's radii (to the tail), the azimuthal limits were $-\pi / 4$ and $\pi / 4$ having midnight as 0 . Each bin had an extension of $6 R_{E}$ and $\pi / 12$ in radial and azimuthal direction, respectively. Each next bin had an overlapping with the previous one in a half step ( $3 R_{E}$ and $\left.\pi / 24\right)$. This was done to improve the statistics so that each bin has at least 10 initial data points and to smoothen the final distribution.

Figure 1 shows the spatial distribution of eddy-diffusion coefficients in GSM coordinate system. As it can be seen, even during the quiet geomagnetic conditions, the values of coefficient vary significantly. It was found that the eddydiffusion generally increases with the distance to the tail 

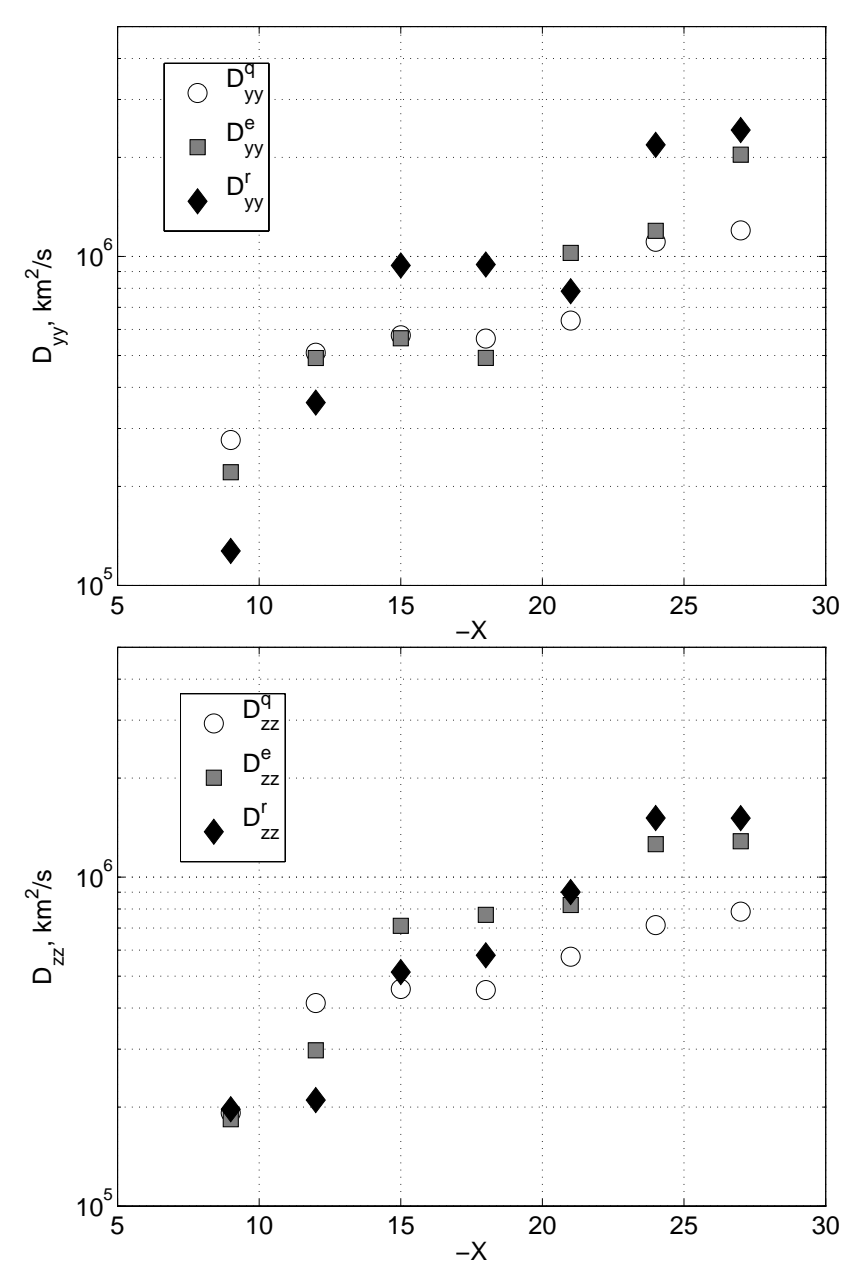

Fig. 2. Variation of eddy-diffusion coefficients with the distance from the Earth, for quiet time (q), expansion (e) and recovery (r) phases.

for all components and all geomagnetic conditions. More detailed analysis shows that during quiet geomagnetic conditions $D_{y y}>D_{z z}$. This fact could be related to the geometry of the plasma sheet, which is much more extended in Y-direction, allowing for an easier development of vortices. The values of eddy diffusion coefficient obtained by us sometimes are higher than the ones typically reported for the plasma sheet (see, for example, Borovsky et al., 1998; Borovsky and Funsten, 2003b; Ovchinnikov et al., 2000; Troshichev et al., 2002; Stepanova et al., 2005). It could be related to the necessity to work with very short series for determination of the root mean square velocities and autocorrelation times, which are related to the quest for acceptable space and time resolution ( $\left.<2 R_{E}, 40 \mathrm{~min}\right)$. Otherwise it would be impossible to discriminate between the expansion and recovery phases. Nevertheless, we are certain that the qualitative behavior is well reproduced by our methodology.
Table 1. Number of values for each data set of eddy-diffusion coefficients.

\begin{tabular}{cccc}
\hline Eddy diffusion component & Quiet & Expansion & Recovery \\
\hline$D_{y y}$ & 5345 & 766 & 922 \\
$D_{z z}$ & 6686 & 1048 & 1227 \\
\hline
\end{tabular}

During substorms Z- and especially Y-components experience an increase, mainly at the distances of $15-25 R_{E}$. During the expansion phase the maximum is more pronounced, during the recovery it starts to spread and to be more irregular in space. Unfortunately Interball-Tail satellite has no measurements at the distances larger than $30 R_{E}$. We can only assume, that eddy diffusion coefficients for both quiet and disturbed conditions will saturate at some value, corresponding to the pure tail configuration of geomagnetic field lines.

Figure 2 shows the variation of eddy diffusion coefficient with the distance from the Earth, obtained by averaging the bins showed in Fig. 1 by the angle. As can easily be seen, both components of the eddy-diffusion coefficients increase with the distance from the Earth for all geomagnetic conditions. Nevertheless, during the expansion phase they are higher by 2 times approximately, especially for the distances $>20 R_{E}$. During the recovery phases both eddy-diffusion components are still high, especially $D_{y y}$. On the average, the eddy diffusion coefficients in Y-direction are higher than that in $Z$ direction. The other feature is: a local minimum for the $D_{y y}$ at $20 R_{E}$, approximately, which is more pronounced during the recovery phase. Nevertheless, our statistics was not good enough to make definite conclusions.

\section{Conclusions}

It was found that even under quiet geomagnetic conditions and in the absence of high speed flows, the plasma in plasma sheet is strongly turbulent. Eddy diffusion coefficients, which determine the turbulent transport, are large and vary in the range starting from $10^{4}$ and occationally reaching the values of $10^{7} \mathrm{~km}^{2} / \mathrm{s}$. Nevertheless, on average, both $D_{y y}$ and $D_{z z}$ components increase in the tailward direction, from $1.9 \times 10^{5} \mathrm{~km}^{2} / \mathrm{s}$ to $7.9 \times 10^{5} \mathrm{~km}^{2} / \mathrm{s}$ in case of $D_{z z}$, and from $2.9 \times 10^{5} \mathrm{~km}^{2} / \mathrm{s}$ to $1.4 \times 10^{6} \mathrm{~km}^{2} / \mathrm{s}$ in case of $D_{y y}$. It is possible that this change in the value of eddy diffusion corresponds to the transition from the region where the presence of dipole geomagnetic field is still notable to the region where the geomagnetic field has the purely tail configuration. These values are higher than average value reported by (Borovsky and Funsten, 2003b) $2.6 \times 10^{5} \mathrm{~km}^{2} / \mathrm{s}$. This fact can be related to the necessity to shorten the bulk velocity series, to be able divide the data into quiet, expansion, and recovery subsets. 
This increase in the values of diffusion coefficients in the tailward direction can be connected to the process of substorm development starting with the first auroral arc brightening at the equatorial boundary of the auroral oval (Akasofu, 1964). During both expansion and recovery phases both $D_{y y}$ and $D_{z z}$ components of the eddy-diffusion coefficients increase most significantly at the distances of $15-25 R_{E}$. It could be related to the increase of reconnection processes in this region. We plan to explore this possibility using the high-resolution data from the Themis mission.

Acknowledgements. The research was supported by the FONDECYT No 1070131 and RFBR grants.

Topical Editor R. Nakamura thanks two anonymous referees for their help in evaluating this paper.

\section{References}

Akasofu, S.-I.: The development of the auroral substorm, Planet. Space Sci., 12(4), 273-282, 1964.

Antonova, E. E. and Ovchinnikov, I. L.: The equilibrium of turbulent current sheet and the current sheet of the Earth's magnetotail, Geomagn. Aeron., 36(1), 7-14, 1996.

Antonova, E. E. and Ovchinnikov, I. L.: Magnetostatically equilibrated plasma sheet with developed medium-scale turbulence: Structure and implications for substorm dynamics, J. Geophys. Res., 104(A8), 17289-17297, 1999.

Antonova, E. E. and Ovchinnikov, I. L.: The model of turbulent plasma sheet during IMF Bz>0, Adv. Space Res., 28(12), 17471752, 2001.

Borovsky, J. E., Elpic, R. C., Funsten, H. O., and Thomsen, M. F.: The Earth's plasma sheet as a laboratory for flow turbulence in high- $\beta$ MHD, J. Plasma Phys., 57(1), 1-34, 1997.

Borovsky, J., Thomsen M., and Elphic, R.: The driving of the plasma sheet by the solar wind, J. Geophys. Res., 103(A8), 17617-17639, 1998.

Borovsky, J. E. and Funsten, H. O.: Role of solar wind turbulence in the coupling of the solar wind to the Earth's magnetosphere, J. Geophys. Res., 108(A6), 1246, doi:10.1029/2002JA009601, 2003a.

Borovsky J. E. and Funsten, H. O.: MHD turbulence in the Earth's plasma sheet: Dynamics, dissipation, and driving, J. Geophys. Res., 108(A7), 1284, doi:10.1029/2002JA009625, 2003 b.

Borovsky, J. E.: MHD Eddy Viscosity: Testing the Concept with the Solar-Wind/Magnetosphere Coupling Data Base, American Physical Society, 47th Annual DPP Meeting, October 24-28, 2005, abstract BI1.004, 2005APS..DPPBI1004B, 2005.
Klimov, S., Romanov, S., Amata, E., et al.: ASPI experiment: measurements of fields and waves on board the INTERBALL1 spacecraft, Ann. Geophys., 15, 514-527, 1997, http://www.ann-geophys.net/15/514/1997/.

Neagu, E., Borovsky, J. E., Thomsen, M. F., Gary, S. P., Baumjohann, W., and Treumann, R. A.: Statistical survey of magnetic field and ion velocity fluctuations in the near-Earth plasma sheet: Active Magnetospheric Particle Trace Explorers/Ion Release Module (AMPTE/IRM) measurements, J. Geophys. Res., 107(A7) 1098, doi:10.1029/2001JA000318, 2002.

Neagu, E., Borovsky, J., Gary, P. S., Jorgensen, A. M., Baumjohann, W., and Treumann, R. A.: Statistical survey of magnetic and velocity fluctuations in the near-Earth plasma sheet: International Sun Earth Explorer (ISEE-2) measurements, J. Geophys. Res., 110, A05203, doi:10.1029/2004JA010448, 2005.

Ovchinnikov, I. L., Antonova, E. E., and Yermolaev, Y. I.: Determination of the turbulent diffusion coefficient in the plasma sheet using the Project INTERBALL data, Kosmicheskiye Issledovaniya (Space Research), 38, 557-561, 2000. (in Russian).

Shiokawa, K., Shinohara, I., Mukai, T., Hayakawa, H., and Cheng, C. Z.: Magnetic field fluctuations during substorm-associated dipolarizations in the nightside plasma sheet around $X=10 \mathrm{R} \mathrm{E}, \mathrm{J}$. Geophys. Res., 110, A05212, doi:10.1029/2004JA010378, 2005.

Stepanova, M. V., Antonova, E. E., Bosqued, J. M., Kovrazhkin, R. A., and Aubel, K. R.: Asymmetry of auroral electron precipitations and its relationship to the substorm expansion phase onset, J. Geophys. Res., 107(A7), 25-1-25-12, doi:10.1029/2001JA003503, 2002.

Stepanova, M., Vucina-Parga, T., Antonova, E., Ovchinnikov, I., and Yermolaev, Y.: Variation of the plasma turbulence in the central plasma sheet during substorm phases observed by the Interball/Tail satellite, J. Atmos. Solar-Terr. Phys., 67, 1815-1820, 2005.

Troshichev, O. A., Antonova, E. E., and Kamide, Y.: Inconsistency of magnetic field and plasma velocity variations in the distant plasma sheet: violation of the "frozen-in" criterion?, Adv. Space Res., 30(12), 2683-2687, 2002.

Yermolaev, Yu. I., Fedorov, A. O., Vaisberg, O. L., Balebanov, V. M., Obod, Yu. A., Jimenez, R., Fleites, J., Llera, L., and Omelchenko, A. N.: Ion distribution dynamics near the Earth's bow shock: first measurements with the 2D ion energy spectrometer CORALL on the INTERBALL/Tail-probe satellite, Ann. Geophys., 15, 533-541, 1997, http://www.ann-geophys.net/15/533/1997/. 